\title{
Borboletas (Lepidoptera, Papilionoidea) em floresta estacional semidecidual do sul do estado de Minas Gerais, Brasil
}

As borboletas desempenham funções essenciais nos ecossistemas, podendo ser utilizadas como bioindicadores. Apesar de sua relevância pouco se conhece sobre o grupo em muitos estados, ecossistemas e unidades de conservação brasileiras, como o estado de Minas Gerais, em que se conhece cerca de 1600 espécies, das quais 25 estão ameaçadas de extinção. Nesse panorama o objetivo do estudo foi inventariar a fauna de borboletas em áreas de floresta estacional semidecidual na região sul de Minas Gerais, Brasil. O trabalho foi conduzido de janeiro de 2016 a maio de 2019, com esforço amostral diferenciado para cada área, totalizando 492 horas amostrais. Os exemplares capturados foram armazenados em envelopes entomológicos e encaminhados para sua identificação no Laboratório de Ecologia e Sistemática de Borboletas (LABBOR), e encontra-se em processo de tombamento na coleção biológica do Laboratório de Zoologia do IFSULDEMINAS, Campus Inconfidentes. Foram coletados 1070 indivíduos, pertencentes a seis famílias e 234 espécies distribuídas em 19 subfamílias. Destaca-se 0 registro da espécie Charonias theano (BOISDUVAL, 1836) ameaçada de extinção, presente na Área de Proteção Ambiental da Bacia Hidrográfica do Rio Machado município de Poço Fundo. O presente estudo evidenciou que a região sul de Minas Gerais abriga uma importante fauna de borboletas, sendo necessário estabelecer ações para conservação dessa biota.

Palavras-chave: Charonias theano; Inventário; Floresta Atlântica; Nymphalidae.

\section{Butterflies (Lepidoptera, Papilionoidea) in a semidecidual station forest in the southern state of Minas Gerais, Brazil}

Butterflies play essential functions in ecosystems, and can be used as bioindicators. Although its relevance, little is known about the group in many Brazilian states, ecosystems and conservation units, like the state of Minas Gerais, where about 1600 species are known, of which 25 are endangered. In this context, the objective of the study was to inventory the butterfly fauna in areas of seasonal semideciduous forest in the southern region of Minas Gerais, Brazil. The work was conducted from January 2016 to May 2019, with a different sampling effort for each area, totaling 492 sample hours. The captured specimens were stored in entomological envelopes and sent for identification at the Laboratory of Ecology and Systematics of Butterflies (LABBOR), and is in the process of being listed in the biological collection of the IFSULDEMINAS Zoology Laboratory, Campus Inconfidentes. 1070 individuals were collected, belonging to six families and 234 species distributed in 19 subfamilies. The record of the species Charonias theano (BOISDUVAL, 1836), which is threatened with extinction, is present in the Environmental Protection Area of the Machado River Basin, in the municipality of Poço Fundo. The present study showed that the southern region of Minas Gerais is home to an important butterfly fauna, and it is necessary to establish actions for the conservation of this biota.

Keywords: Charonias theano; Inventory; Atlantic forest; Nymphalidae.

Topic: Conservação da Biodiversidade

Reviewed anonymously in the process of blind peer
Received: $\mathbf{1 1 / 0 3 / 2 0 2 0}$

Approved: 10/04/2020
Lucas Ramos Vieira (iD)

Instituto Federal do Sul de Minas, Brasil

http://lattes.cnpq.br/3900496640397792

http://orcid.org/0000-0002-8128-875X

lucas.ramos@alunos.ifsuldeminas.edu.br

Lauana Daló da Silva (ii

Instituto Federal do Sul de Minas, Brasil http://lattes.cnpq.br/3994276254064018 http://orcid.org/0000-0002-6549-2259

lauana.dalo@gmail.com

Luan de Andrade Oliveira

Instituto Federal do Sul de Minas, Brasil

http://lattes.cnpq.br/2070511132058997

http://orcid.org/0000-0002-2602-3839

luanoliveirabioif@hotmail.com

d

DOI: 10.6008/CBPC2318-2881.2020.002.0002

\author{
Augusto Henrique Batista Rosa (ID) \\ Universidade Estadual de Campinas, Brasil \\ http://lattes.cnpq.br/4503377815275894 \\ http://orcid.org/0000-0002-0151-114X \\ augustohbrosa@hotmail.com \\ Marcos Magalhães de Souza (iD \\ Universidade Federal de Lavras, Brasil \\ http://lattes.cnpq.br/2334845279402555 \\ http://orcid.org/0000-0003-0415-1714 \\ marcos.souza@ifsuldeminas.edu.br
}

Referencing this:

VIEIRA, L. R.; SILVA, L. D.; OLIVEIRA, L. A.; ROSA, A. H. B.; SOUZA, M. M.. Borboletas (lepidoptera, papilionoidea) em floresta estacional semidecidual do sul do estado de Minas Gerais, Brasil. Nature and Conservation, v.13, n.2, p.14-25, 2020. DOI:

http://doi.org/10.6008/CBPC2318-2881.2020.002.0002 


\section{INTRODUÇÃO}

A ordem Lepidoptera reúne os insetos popularmente conhecidos como borboletas e mariposas, formando a segunda maior ordem de insetos, sendo que no Brasil são encontradas cerca de 12.735 espécies, em que 3.465 são borboletas (CASAGRANDE et al., 2019). Atualmente esses insetos estão agrupados em uma única superfamília, Papilionoidea, distribuídos em sete famílias: Hesperiidae, Lycaenidae, Nymphalidae, Papilionidae, Pieridae, Riodinidae e Hedylidae (MITTER et al., 2017).

As borboletas desempenham funções essenciais no ecossistema, como polinização, herbivoria e decomposição (LOMOV et al., 2006), e são consideradas indicadores de ambiente e de diversidade (FREITAS et al., 2006). Apesar dessa relevância, o conhecimento básico como inventários de espécies ainda é deficiente em muitos estados, ecossistemas e unidades de conservação no Brasil, como por exemplo o estado de Minas Gerais, que mesmo nessa condição, se conhece cerca de 1.600 espécies de borboletas, das quais 25 estão ameaçadas de extinção (CASAGRANDE et al., 1998; COPAM, 2010; ICMBIO, 2018). Esse estado apresenta diferentes fitofisionomias de biomas distintos, com destaque para a floresta estacional semidecidual, de domínio da Mata Atlântica, que perfaz a maior cobertura vegetal do estado (OLIVEIRA FILHO, 2006).

Alguns inventário de borboletas foram realizados em diferentes biomas de Minas Gerais, como o Cerrado próximo aos municípios de Belo Horizonte, Curvelo, Paraopeba, Paracatu (BROWN JUNIOR et al., 1967a; 1967b; 1968; BROWN JUNIOR, 1972a), no Parque Estadual Serra do Intendente (NERY et al., 2014), na Serra do Cipó (PIRES et al., 2018) e na Área de Proteção Ambiental Serra de São José (HENRIQUES et al., 2019); em Mata Atlântica, nos municípios de Itamonte, Passa Quatro e Virgínia (ZIKÁN et al., 1968), Poços de Caldas (EBERT, 1969), em Muzambinho (ANDRADE et al., 2017), Baependi (Oliveira et al., 2018); além de áreas urbanas (SILVA et al., 2007; SOARES et al., 2012; ARAÚJO et al., 2015) e em áreas de ecotono entre os dois biomas, na Área de Proteção Especial Manancial Cercadinho (SILVA et al., 2012) e Parque Estadual Serra do Rola Moça (SOLDATI et al., 2019).

Considerando o número de espécies em outros estudos realizados em floresta estacional semidecidual (SILVA et al., 2007; SILVA et al., 2017) espera-se registrar cerca de 300 espécies, obtendo maior riqueza em áreas inseridas em unidades de conservação. Nessa perspectiva, esse estudo teve o objetivo de inventariar a fauna de borboletas em áreas de floresta estacional semidecidual na região sul do estado de Minas Gerais, sudeste do Brasil.

\section{MATERIAIS E MÉTODOS}

Foram realizadas coletas em diferentes localidades do sul do estado de Minas Gerais, Brasil, em áreas que predominam floresta estacional semidecidual, fitofisionomia de domínio da Mata Atlântica (OLIVEIRA FILHO, 2006), associados a áreas agrícolas e de pastagem. O esforço de amostragem foi diferenciado entre as localidades em função de logística (Tabela 1).

Nos municípios de Bueno Brandão, Inconfidentes e Ouro Fino as amostragens foram realizadas quinzenalmente com esforço amostral diário de quatro horas, no período de janeiro de 2016 a maio de 
2019. Na Área de Proteção Ambiental da Bacia Hidrográfica do Rio Machado (APA do Rio Machado) foram realizadas oito campanhas, concentrando o esforço amostral nos municípios de Fama, Machado e Poço Fundo, de dois a três dias consecutivos por mês, no período de setembro de 2018 a abril de 2019, com esforço de 8 horas diárias. Essa unidade de conservação sofre diferentes ações antrópicas como desmatamento, pecuária, agricultura e urbanização. Nos demais municípios o esforço amostral ocorreu de forma esporádica.

Tabela 1: Locais amostrados e esforço amostral para registro de borboletas em florestas estacionais semideciduais no sul do estado de Minas Gerais.

\begin{tabular}{|l|l|l|}
\hline Município & Coordenadas & Esforço amostral (dia e horas) \\
\hline Bueno Brandão & $22^{\circ} 26^{\prime} 27^{\prime \prime} \mathrm{S}, 46^{\circ} 21^{\prime} 6^{\prime \prime} \mathrm{W}$ & 27 dias, 108 horas \\
\hline Congonhal & $22^{\circ} 09^{\prime} 10^{\prime \prime} \mathrm{S}, 46^{\circ} 02^{\prime} 22^{\prime \prime} \mathrm{W}$ & 1 dia, 4 horas \\
\hline Fama & $21^{\circ} 24^{\prime} 21^{\prime \prime} \mathrm{S}, 45^{\circ} 49^{\prime} 44^{\prime \prime} \mathrm{W}$ & 6 dias, 48 horas \\
\hline Inconfidentes & $22^{\circ} 19^{\prime} 2^{\prime \prime} \mathrm{S}, 46^{\circ} 19^{\prime} 42^{\prime \prime} \mathrm{W}$ & 45 dias, 180 horas \\
\hline Itajubá & $22^{\circ} 25^{\prime} 32^{\prime \prime} \mathrm{S}, 45^{\circ} 27^{\prime} 10^{\prime \prime} \mathrm{W}$ & 1 dia, 4 horas \\
\hline Jacutinga & $22^{\circ} 17^{\prime} 08^{\prime \prime} \mathrm{S}, 46^{\circ} 36^{\prime} 44^{\prime \prime} \mathrm{W}$ & 1 dia, 4 horas \\
\hline Machado & $21^{\circ} 40^{\prime} 30^{\prime \prime} \mathrm{S}, 45^{\circ} 55^{\prime} 12^{\prime \prime} \mathrm{W}$ & 6 dias, 48 horas \\
\hline Ouro Fino & $22^{\circ} 16^{\prime} 59^{\prime \prime} \mathrm{S}, 46^{\circ} 22^{\prime} 08^{\prime \prime} \mathrm{W}$ & 10 dias, 40 horas \\
\hline Olímpio Noronha & $22^{\circ} 04^{\prime} 04^{\prime \prime} \mathrm{S}, 45^{\circ} 15^{\prime} 50^{\prime \prime} \mathrm{W}$ & 1 dia, 4 horas \\
\hline Pouso Alegre & $22^{\circ} 13^{\prime} 48^{\prime \prime} \mathrm{S}, 45^{\circ} 56^{\prime} 11^{\prime \prime} \mathrm{W}$ & 1 dia, 4 horas \\
\hline Poço Fundo & $21^{\circ} 46^{\prime} 51^{\prime \prime} \mathrm{S}, 45^{\circ} 57^{\prime} 54^{\prime \prime} \mathrm{W}$ & 6 dias, 48 horas \\
\hline Total & & 105 dias, 492 horas \\
\hline
\end{tabular}

Os adultos de borboletas foram coletados por busca ativa, com uso de redes entomológicas, e posteriormente sacrificados, com pressão no tórax, e armazenados individualmente em envelopes entomológicos com informações de local, data e coletor (Licença SISBIO 63914-1 e IEF/MG 062/2018).

Os espécimes coletados foram identificados por especialistas do Laboratório de Ecologia e Sistemática de Borboletas (LABBOR), na Universidade Estadual de Campinas (UNICAMP) e com auxílio da lista ilustrada de borboletas Americanas (WARREN et al., 2017). A taxonomia a nível de espécie/subespécie segue Lamas (2004) com algumas atualizações taxômicas de trabalhos posteriores a essa literatura. A taxonomia a nível de subfamílias de Nymphalidae segue Wahlberg et al. (2009), para Hesperiidae segue Mielke (2005a; 2005b; 2014a; 2014b; 2014c), para Riodinidae segue Seraphim et al. (2018) e para as demais famílias segue Lamas (2004). O material encontra se depositado, parte na Coleção de Borboleta e Mariposas do Museu de Zoologia da Universidade Estadual de Campinas (ZUEC-LEP UNICAMP), e parte na coleção do Laboratório de Zoologia, do Instituto Federal de Educação, Ciências e Tecnologia do Sul de Minas (IFSULDEMINAS) - Campus Inconfidentes. Para avaliar a eficiência de coleta foi utilizado os estimadores de espécies (Jackknife 1), com o uso do programa Software R (R Development Core Team), com 1000 reamostragens.

\section{RESULTADOS}

Foram coletados 1.070 indivíduos de 234 espécies, distribuídas em 19 subfamílias de seis famílias de borboletas, sendo Nymphalidae de maior riqueza (137 espécies, 59\% de todas as espécies amostradas), seguida por Hesperiidae (28 espécies, 12\%), Riodinidae (24 espécies, 10,2\%), Pieridae (22 espécies, 9,4\%), Papilionidae (12 espécies, 5,1\%) e Lycaenidae (11 espécies, 4,7\%). 
A espécie mais abundante foi Mechanitis polymnia casabranca (HAENSCH, 1905) (Figura 1), subfamília Danainae, Nymphalidae, com 34 indivíduos (3\%). Já a espécie Tegosa claudina (ESCHSCHOLTZ, 1821), subfamilia Nymphalinae, Nymphalidae, foi considerada a mais frequente, estando presente em 23 dias de amostragem (27\%).

Na APA do Rio Machado (municípios de Fama, Machado e Poço Fundo) foram registradas 162 espécies (Tabela 2), o que representam cerca de 70,1\% das espécies do presente estudo, com destaque para Charonias theano (BOISDUVAL, 1836), uma espécie ameaçada de extinção (Figura 2).

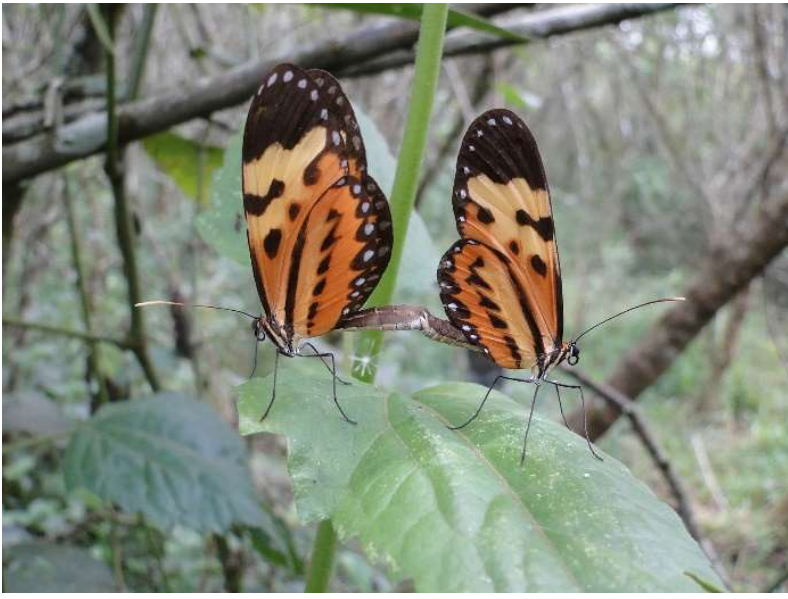

Figura 1: Mechanitis polymnia casabranca (Haensch, 1905) coletada na APA da bacia do Rio Machado, sul de Minas Gerais, Brasil.

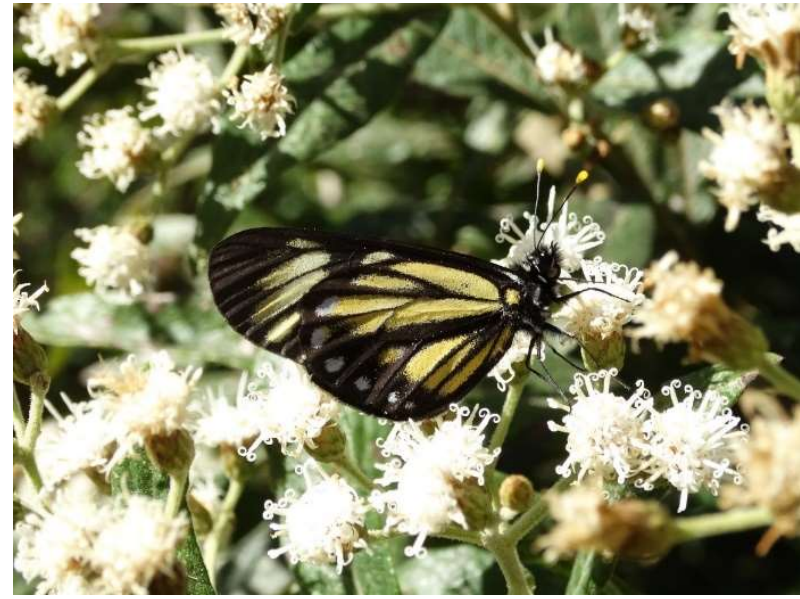

Figura 2: Charonias theano (BOISDUVAL, 1836), espécie ameaçada de extinção na categoria "Em Perigo", coletada na APA da bacia do Rio Machado, sul de Minas Gerais, Brasil.

Tabela 2: Lista de borboletas coletadas em diferentes em florestas estacionais semideciduais em diferentes municípios do sul de Minas Gerais, sudeste do Brasil. BB = Bueno Brandão; $C O=$ Congonhal; FA = Fama; IN = Inconfidentes; ITA = Itajubá; JA = Jacutinga; $\mathrm{MA}=$ Machado; OF = Ouro fino; ON = Olímpio Noronha; $\mathrm{PA}=\mathrm{Pouso}$ Alegre; PF = Poço Fundo. $\mathrm{S}=$ Número de espécies.

\begin{tabular}{|c|c|c|c|c|c|c|c|c|c|c|c|}
\hline \multirow[t]{2}{*}{ Taxa } & \multicolumn{11}{|c|}{ Municípios } \\
\hline & BB & $\mathrm{CO}$ & FA & IN & ITA & JA & MA & OF & ON & PA & PF \\
\hline \multicolumn{12}{|l|}{ HESPERIIDAE (28) } \\
\hline \multicolumn{12}{|l|}{ Hesperiinae (4) } \\
\hline Anthoptus epictetus (FABRICIUS, 1793) & $\mathrm{x}$ & - & - & - & - & - & - & - & - & - & - \\
\hline Eutychide physcella (HEWITSON, 1866) & - & - & - & - & - & - & - & - & - & - & $x$ \\
\hline Hylephila phyleus (DRURY, 1773) & - & - & - & $\mathrm{x}$ & - & - & - & - & - & - & - \\
\hline Vehilius clavicula (PLÖTZ, 1884) & $\mathrm{x}$ & - & - & - & - & - & - & - & - & - & - \\
\hline \multicolumn{12}{|l|}{ Pyrginae (23) } \\
\hline Achlyodes busirus rioja EVANS, 1953 & - & - & - & - & - & - & - & - & - & - & $\mathrm{X}$ \\
\hline Astraptes anaphus anaphus (CRAMER, 1777) & - & - & - & - & - & - & - & $\mathrm{x}$ & - & - & - \\
\hline Astraptes sp. & - & - & - & - & - & - & - & $\mathrm{X}$ & - & - & - \\
\hline Autochton sp. & - & - & - & - & - & - & $\mathrm{x}$ & - & - & - & $\mathrm{x}$ \\
\hline Chioides catillus catillus (CRAMER, 1779) & $\mathrm{X}$ & - & - & $\mathrm{X}$ & - & - & - & - & - & - & - \\
\hline Eantis thraso (HÜBNER, [1807]) & - & - & $\mathrm{x}$ & - & - & - & - & - & - & - & $\mathrm{x}$ \\
\hline Gorgythion begga begga (PRITTWITZ, 1868) & - & - & - & $\mathrm{x}$ & - & - & - & $\mathrm{x}$ & - & - & - \\
\hline Heliopetes alana (REAKIRT, 1868) & - & - & - & - & - & - & - & - & - & - & $\mathrm{x}$ \\
\hline Heliopetes arsalte (LINNAEUS, 1758) & - & - & $\mathrm{X}$ & $\mathrm{x}$ & - & - & - & - & - & - & - \\
\hline Heliopetes omrina (BUTLER, 1870) & $\mathrm{x}$ & - & - & $\mathrm{X}$ & - & - & - & - & - & - & - \\
\hline Heliopyrgus domicella willi (PLÖTZ, 1884) & - & - & $\mathrm{x}$ & - & - & - & - & - & - & - & - \\
\hline Pyrgus orcus (STOLL, 1780) & - & - & $\mathrm{x}$ & $\mathrm{x}$ & - & - & $\mathrm{x}$ & $\mathrm{x}$ & - & $\mathrm{X}$ & $\mathrm{x}$ \\
\hline Pythonides herennius lusorius (MABILLE, 1891) & - & - & $\mathrm{x}$ & - & - & - & - & - & - & - & - \\
\hline Pythonides jovianus fabricii KIRBY, 1871 & - & - & $\mathrm{x}$ & - & - & - & - & - & - & - & $\mathrm{x}$ \\
\hline Pythonides lancea (HEWITSON, 1868) & - & - & - & $\mathrm{x}$ & - & - & - & $\mathrm{x}$ & - & - & - \\
\hline Quadrus cerialis (STOLL, 1782) & - & - & - & - & - & - & - & - & - & - & $\mathrm{x}$ \\
\hline Theagenes dichrous (MABILLE, 1878) & - & - & $x$ & - & - & - & - & - & - & - & - \\
\hline Trina geometrina geometrina (FELDER \& FELDER, 1867) & - & - & $\mathrm{X}$ & $\mathrm{x}$ & - & - & $\mathrm{X}$ & - & - & - & - \\
\hline
\end{tabular}




\begin{tabular}{|c|c|c|c|c|c|c|c|c|c|c|c|}
\hline Urbanus dorantes dorantes (STOLL, 1790) & - & - & $\mathrm{X}$ & - & - & - & $\mathrm{x}$ & - & - & - & $\mathrm{x}$ \\
\hline Urbanus procne (PLÖTZ, 1881) & - & - & $\mathrm{X}$ & $\mathrm{x}$ & - & - & - & - & - & - & - \\
\hline Urbanus simplicius (STOLL, 1790) & - & - & $\mathrm{X}$ & $x$ & - & - & - & - & - & - & $\mathrm{x}$ \\
\hline Urbanus teleus (HÜBNER, 1821) & - & - & $x$ & - & - & - & - & - & - & - & $\mathrm{x}$ \\
\hline Xenophanes tryxus (STOLL, 1780) & - & - & - & $\mathrm{x}$ & - & - & - & - & - & - & $\mathrm{X}$ \\
\hline \multicolumn{12}{|l|}{ Pyrrhopyginae (1) } \\
\hline Pyrrhopyge sp. & - & - & - & - & - & - & $\mathrm{x}$ & - & - & - & - \\
\hline \multicolumn{12}{|l|}{ LYCAENIDAE (11) } \\
\hline \multicolumn{12}{|l|}{ Polyommatinae (2) } \\
\hline Hemiargus hanno hanno (STOLL, 1790) & - & - & $\mathrm{X}$ & - & - & - & $\mathrm{x}$ & - & - & - & $\mathrm{X}$ \\
\hline Leptotes cassius cassius (CRAMER, 1775) & - & - & - & $\mathrm{x}$ & - & $\mathrm{x}$ & $\mathrm{x}$ & - & - & - & $\mathrm{x}$ \\
\hline \multicolumn{12}{|l|}{ Theclinae (9) } \\
\hline Arawacus meliboeus (FABRICIUS, 1793) & $\mathrm{x}$ & - & $\mathrm{X}$ & - & - & - & - & $x$ & - & - & $\mathrm{X}$ \\
\hline Calycopis bellera (HEWITSON, 1877) & - & - & - & $x$ & - & - & - & - & - & - & - \\
\hline Calycopis sp. & - & - & - & - & - & - & - & - & - & - & $\mathrm{X}$ \\
\hline Evenus latreillii (HEWITSON, 1865) & - & - & - & - & - & - & - & - & - & - & $\mathrm{X}$ \\
\hline Laothus phydela (HEWITSON, 1867) & - & - & - & $x$ & - & - & - & $\mathrm{x}$ & - & - & - \\
\hline Pseudolycaena marsyas (LINNAEUS, 1758) & $\mathrm{X}$ & - & - & $\mathrm{x}$ & - & - & - & - & - & - & - \\
\hline Strephonota sphinx (FABRICIUS, 1775) & $\mathrm{x}$ & - & - & - & - & - & - & - & - & - & - \\
\hline Strymon cestri (REAKIRT, [1867]) & - & - & - & - & - & - & - & - & - & - & $\mathrm{x}$ \\
\hline Strymon sp. & - & - & - & $x$ & - & - & - & - & - & - & $\mathrm{X}$ \\
\hline \multicolumn{12}{|l|}{ NYMPHALIDAE (137) } \\
\hline \multicolumn{12}{|l|}{ Apaturinae (4) } \\
\hline Doxocopa kallina (STAUDINGER, 1886) & - & - & - & $\mathrm{x}$ & - & - & - & - & - & - & $\mathrm{x}$ \\
\hline Doxocopa laurentia laurentia (GODART, [1824]) & - & - & - & $\mathrm{x}$ & - & - & - & - & - & - & $\mathrm{X}$ \\
\hline Doxocopa linda mileta (BOISDUVAL, 1870) & - & - & - & $\mathrm{X}$ & - & - & - & - & - & - & $\mathrm{X}$ \\
\hline Doxocopa zunilda zunilda (GODART, [1824]) & - & - & $\mathrm{X}$ & - & - & - & - & - & - & - & - \\
\hline \multicolumn{12}{|l|}{ Biblidinae (28) } \\
\hline Biblis hyperia nectanabis (FRUHSTORFER, 1909) & $\mathrm{X}$ & - & $\mathrm{X}$ & $\mathrm{x}$ & - & - & - & $\mathrm{x}$ & - & - & - \\
\hline Callicore hydaspes (DRURY, 1782) & - & - & - & - & - & - & $\mathrm{x}$ & - & - & - & - \\
\hline Callicore sorana sorana (GODART, [1824]) & - & - & - & $\mathrm{x}$ & - & - & - & $\mathrm{x}$ & - & - & - \\
\hline Catonephele sabrina (HEWITSON, 1852) & - & - & - & - & - & - & - & - & - & - & $\mathrm{x}$ \\
\hline Cybdelis phaesyla (HÜBNER, [1831]) & - & - & - & - & - & - & - & - & - & - & $\mathrm{X}$ \\
\hline Diaethria candrena candrena (GODART, [1824]) & - & - & $\mathrm{X}$ & $\mathrm{X}$ & - & - & $\mathrm{x}$ & $\mathrm{x}$ & - & - & - \\
\hline Diaethria clymena janeira (FELDER, 1862) & - & - & $\mathrm{X}$ & $\mathrm{x}$ & $\mathrm{X}$ & - & - & - & - & - & - \\
\hline Diaethria eluina eluina (HEWITSON, [1855]) & $\mathrm{X}$ & - & $\mathrm{X}$ & $\mathrm{x}$ & - & - & - & $\mathrm{x}$ & - & - & $\mathrm{x}$ \\
\hline Dynamine aerata (BUTLER, 1877) & - & - & - & $x$ & - & - & - & - & - & - & - \\
\hline Dynamine agacles agacles (DALMAN, 1823) & - & - & - & $\mathrm{x}$ & - & - & - & - & - & - & - \\
\hline Dynamine artemisia artemisia (FABRICIUS, 1793) & - & - & - & $x$ & - & - & - & - & - & - & $\mathrm{x}$ \\
\hline Dynamine postverta postverta (CRAMER, 1779) & - & - & - & $\mathrm{x}$ & - & - & - & - & - & - & - \\
\hline Dynamine tithia tithia (HÜBNER, [1823]) & - & - & - & $x$ & - & - & $\mathrm{x}$ & - & - & - & $\mathrm{X}$ \\
\hline Ectima thecla thecla (FABRICIUS, 1796) & $\mathrm{X}$ & - & - & $\mathrm{X}$ & - & - & - & - & - & - & $\mathrm{X}$ \\
\hline Epiphile hubneri (HEWITSON, 1861) & - & - & - & - & - & - & - & - & - & - & $\mathrm{x}$ \\
\hline Epiphile orea orea (HÜBNER, [1823]) & - & - & - & $x$ & - & - & - & - & - & - & $\mathrm{x}$ \\
\hline Eunica eburnea (FRUHSTORFER, 1907) & - & - & - & - & - & - & - & $\mathrm{x}$ & - & - & $\mathrm{x}$ \\
\hline Eunica margarita (GODART, [1824]) & - & - & - & $\mathrm{x}$ & - & - & $\mathrm{x}$ & - & - & - & - \\
\hline Eunica tatila bellaria (FRUHSTORFER, 1908) & - & - & - & $\mathrm{x}$ & - & - & - & - & - & - & - \\
\hline Haematera pyrame pyrame (HÜBNER, [1819]) & - & - & - & - & - & - & $\mathrm{x}$ & - & - & - & $\mathrm{x}$ \\
\hline Hamadryas amphinome amphinome (LINNAEUS, 1767) & - & - & $\mathrm{X}$ & - & - & - & - & - & - & - & - \\
\hline Hamadryas epinome (FELDER \& FELDER, 1867) & $\mathrm{X}$ & - & - & $\mathrm{X}$ & - & $\mathrm{X}$ & $\mathrm{x}$ & - & - & - & - \\
\hline Hamadryas februa februa (HÜBNER, [1823]) & - & - & $\mathrm{X}$ & $\mathrm{x}$ & - & - & - & - & - & $\mathrm{x}$ & $\mathrm{x}$ \\
\hline Hamadryas feronia feronia (LINNAEUS, 1758) & - & - & - & $\mathrm{x}$ & $\mathrm{X}$ & - & - & - & - & - & - \\
\hline Hamadryas fornax fornax (HÜBNER, [1823]) & - & - & - & - & $\mathrm{X}$ & - & - & - & - & - & - \\
\hline Mestra hersilia apicalis (STAUDINGER, 1886) & - & - & $\mathrm{X}$ & - & - & - & - & - & - & - & - \\
\hline Myscelia orsis (DRURY, 1782) & - & - & - & $x$ & - & - & - & - & - & - & $\mathrm{x}$ \\
\hline Pyrrhogyra neaerea (LINNAEUS, 1758) & - & - & - & - & - & - & $\mathrm{x}$ & - & - & - & - \\
\hline \multicolumn{12}{|l|}{ Charaxinae (8) } \\
\hline Fountainea ryphea phidile (GEYER, 1837) & $\mathrm{X}$ & - & - & $\mathrm{X}$ & - & - & - & - & - & - & - \\
\hline Hypna clytemnestra huebneri (BUTLER, 1866) & $\mathrm{X}$ & - & - & $\mathrm{x}$ & - & - & - & $\mathrm{x}$ & - & - & $\mathrm{x}$ \\
\hline Memphis acidalia victoria (DRUCE, 1877) & - & - & - & $\mathrm{x}$ & - & - & - & - & - & - & - \\
\hline Memphis appias (HÜBNER, [1825]) & - & - & - & - & - & - & - & $\mathrm{x}$ & - & - & - \\
\hline Memphis arginussa arginussa (GEYER, 1832) & - & - & - & - & - & - & - & $\mathrm{x}$ & - & - & - \\
\hline Memphis moruus stheno (PRITTWITZ, 1865) & - & - & - & - & - & - & - & - & - & - & $\mathrm{x}$ \\
\hline Memphis otrere (HÜBNER, [1825]) & - & - & - & - & - & - & - & - & - & - & $\mathrm{x}$ \\
\hline Zaretis strigosus (GMELIN, [1790]) & - & - & - & $\mathrm{X}$ & - & - & - & - & - & - & - \\
\hline Danainae (26) & & & & & & & & & & & \\
\hline
\end{tabular}




\begin{tabular}{|c|c|c|c|c|c|c|c|c|c|c|c|}
\hline Aeria olena (WEYMER, 1875) & $\mathrm{x}$ & - & $\mathrm{x}$ & $\mathrm{X}$ & - & $x$ & - & $\mathrm{x}$ & - & - & $x$ \\
\hline Brevioleria aelia plisthenes (d'ALMEIDA, 1958) & - & - & - & - & - & - & - & - & - & - & $x$ \\
\hline Danaus erippus (CRAMER, 1775) & $\mathrm{x}$ & $\mathrm{x}$ & - & $\mathrm{X}$ & - & - & - & - & - & - & $x$ \\
\hline Danaus gilippus gilippus (CRAMER, 1775) & $\mathrm{X}$ & - & $\mathrm{X}$ & $\mathrm{X}$ & - & - & $\mathrm{X}$ & - & - & - & $\mathrm{x}$ \\
\hline Dircenna dero (HÜBNER, 1823) & $\mathrm{x}$ & - & - & $\mathrm{X}$ & - & - & $\mathrm{x}$ & $\mathrm{X}$ & - & $\mathrm{X}$ & $\mathrm{X}$ \\
\hline Episcada carcinia SCHAUS, 1902 & - & - & - & $\mathrm{X}$ & - & - & - & - & - & - & - \\
\hline Episcada hymenaea hymenaea (PRITTWITZ, 1865) & $\mathrm{X}$ & - & - & $\mathrm{x}$ & - & - & - & $\mathrm{X}$ & - & - & $\mathrm{x}$ \\
\hline Episcada striposis (HAENSCH, 1909) & - & - & - & - & - & - & - & - & - & - & $\mathrm{x}$ \\
\hline Epityches eupompe (GEYER, 1832) & - & - & - & - & - & - & $\mathrm{X}$ & $\mathrm{X}$ & - & - & - \\
\hline Hypoleria alema proxima (WEYMER, 1899) & - & - & - & - & - & - & $\mathrm{x}$ & - & - & - & $\mathrm{x}$ \\
\hline Hypothyris euclea laphria (DOUBLEDAY, 1847) & $\mathrm{X}$ & - & $\mathrm{x}$ & $\mathrm{x}$ & - & - & - & $\mathrm{X}$ & - & - & - \\
\hline Hypothyris ninonia daeta (BOISDUVAL, 1836) & $x$ & - & $\mathrm{X}$ & $\mathrm{X}$ & - & - & $\mathrm{X}$ & $\mathrm{x}$ & - & - & $\mathrm{x}$ \\
\hline Ithomia agnosia zikani (D'ALMEIDA, 1940) & - & - & - & $\mathrm{x}$ & - & - & - & $\mathrm{x}$ & - & $\mathrm{X}$ & $\mathrm{x}$ \\
\hline Ithomia drymo (HÜBNER, 1816) & $x$ & - & - & - & - & - & - & $x$ & - & - & - \\
\hline Lycorea halia discreta (HAENSCH, 1909) & $x$ & - & - & $\mathrm{x}$ & - & - & - & - & - & - & - \\
\hline Mcclungia cymo salonina (HEWITSON, 1855) & $\mathrm{X}$ & - & $\mathrm{X}$ & $\mathrm{x}$ & - & - & $\mathrm{x}$ & $\mathrm{x}$ & - & - & $\mathrm{X}$ \\
\hline Mechanitis lysimnia lysimnia (FABRICIUS, 1793) & $\mathrm{X}$ & - & $\mathrm{X}$ & $\mathrm{X}$ & - & - & - & $\mathrm{X}$ & - & - & $\mathrm{x}$ \\
\hline Mechanitis polymnia casabranca (HAENSCH, 1905) & $\mathrm{X}$ & - & $\mathrm{x}$ & $\mathrm{x}$ & - & - & $\mathrm{x}$ & - & - & $\mathrm{X}$ & $\mathrm{x}$ \\
\hline Melinaea ethra (GODART, 1819) & - & - & $\mathrm{x}$ & - & - & - & - & - & - & - & - \\
\hline Methona themisto (HÜBNER, 1818) & - & - & - & $\mathrm{X}$ & - & - & - & - & - & - & - \\
\hline Oleria aquata (WEYMER, 1875) & $\mathrm{x}$ & - & - & - & - & - & - & - & - & - & - \\
\hline Placidina euryanassa (FELDER \& FELDER, 1860) & - & - & $\mathrm{X}$ & $\mathrm{x}$ & - & - & - & $\mathrm{X}$ & - & - & $\mathrm{X}$ \\
\hline Pseudoscada acilla quadrifasciata TALBOT, 1928 & - & - & - & - & - & - & $\mathrm{x}$ & - & - & - & - \\
\hline Pseudoscada erruca (HEWITSON, 1855) & - & - & - & $\mathrm{x}$ & - & - & - & - & - & - & - \\
\hline Pteronymia carlia (SCHAUS, 1902) & - & - & - & $\mathrm{x}$ & - & - & - & - & - & - & $\mathrm{x}$ \\
\hline Thyridia psidii hippodamia (FABRICIUS, 1775) & $\mathrm{x}$ & - & - & $\mathrm{x}$ & - & - & - & - & - & - & - \\
\hline \multicolumn{12}{|l|}{ Heliconiinae (16) } \\
\hline Actinote canutia (HOPFFER, 1874) & $x$ & - & $\mathrm{X}$ & $\mathrm{X}$ & - & - & $\mathrm{X}$ & - & - & - & $\mathrm{x}$ \\
\hline Actinote genitrix genitrix d'ALMEIDA, 1922 & - & - & - & - & - & - & - & - & - & - & $\mathrm{X}$ \\
\hline Actinote pellenea pellenea (HÜBNER, [1821]) & - & - & - & $\mathrm{x}$ & - & - & - & - & - & - & - \\
\hline Actinote pyrrha pyrrha (FABRICIUS, 1775) & $\mathrm{x}$ & $\mathrm{x}$ & $\mathrm{x}$ & $\mathrm{x}$ & - & - & $\mathrm{x}$ & $\mathrm{X}$ & - & - & $\mathrm{x}$ \\
\hline Actinote sp. 1 & $\mathrm{x}$ & - & - & $\mathrm{x}$ & - & - & $\mathrm{x}$ & $\mathrm{X}$ & - & - & $\mathrm{x}$ \\
\hline Actinote sp. 2 & - & - & - & $\mathrm{X}$ & - & - & - & - & - & - & - \\
\hline Agraulis vanillae maculosa (STICHEL, [1908]) & $\mathrm{x}$ & - & $\mathrm{X}$ & $\mathrm{x}$ & - & $\mathrm{X}$ & - & - & - & $\mathrm{X}$ & $\mathrm{x}$ \\
\hline Dione juno juno (CRAMER, 1779) & $x$ & - & - & $\mathrm{x}$ & - & - & - & - & - & - & - \\
\hline Dryadula phaetusa (LINNAEUS, 1758) & - & - & - & $\mathrm{X}$ & - & - & - & $\mathrm{X}$ & - & - & - \\
\hline Dryas iulia alcionea (CRAMER, 1779) & $x$ & - & $\mathrm{x}$ & $\mathrm{x}$ & - & - & - & - & - & - & $x$ \\
\hline Eueides aliphera aliphera (GODART, 1819) & $x$ & - & - & $\mathrm{x}$ & - & - & - & - & - & - & - \\
\hline Eueides isabella dianasa (HÜBNER, [1806]) & - & - & - & - & - & - & - & - & - & $\mathrm{x}$ & $x$ \\
\hline Heliconius besckei (MÉNÉTRIÉS, 1857) & - & - & $\mathrm{X}$ & $\mathrm{x}$ & - & - & - & - & $\mathrm{X}$ & - & $\mathrm{x}$ \\
\hline Heliconius erato phyllis (FABRICIUS, 1775) & $\mathrm{x}$ & - & $\mathrm{X}$ & $\mathrm{x}$ & - & - & $\mathrm{X}$ & $\mathrm{X}$ & $\mathrm{X}$ & $\mathrm{X}$ & $\mathrm{x}$ \\
\hline Heliconius ethilla narcaea (GODART, 1819) & $x$ & - & - & $\mathrm{x}$ & - & - & $\mathrm{X}$ & $\mathrm{x}$ & - & - & $\mathrm{x}$ \\
\hline Philaethria wernickei (RÖBER, 1906) & $x$ & - & - & - & - & - & - & - & - & - & - \\
\hline \multicolumn{12}{|l|}{ Limenitidinae (6) } \\
\hline Adelpha abia (HEWITSON, 1850) & - & - & - & - & - & - & $\mathrm{x}$ & - & - & - & $\mathrm{x}$ \\
\hline Adelpha calliphane (FRUHSTORFER, 1915) & - & - & $\mathrm{x}$ & - & - & - & - & $\mathrm{X}$ & - & - & - \\
\hline Adelpha falcipennis (FRUHSTORFER, 1915) & - & - & $\mathrm{x}$ & - & - & - & - & - & - & - & - \\
\hline Adelpha mythra (GODART, [1824]) & - & - & - & - & - & - & - & - & - & - & $\mathrm{x}$ \\
\hline Adelpha syma (GODART, [1824]) & $\mathrm{X}$ & - & $\mathrm{X}$ & $\mathrm{x}$ & - & - & - & $\mathrm{X}$ & - & - & $\mathrm{x}$ \\
\hline Adelpha thessalia indefecta FRUHSTORFER, 1913 & - & - & - & $\mathrm{X}$ & - & - & - & - & - & - & - \\
\hline \multicolumn{12}{|l|}{ Nymphalinae (17) } \\
\hline Anartia amathea roeselia (ESCHSCHOLTZ, 1821) & $\mathrm{X}$ & - & $\mathrm{X}$ & $\mathrm{X}$ & - & - & $\mathrm{X}$ & $\mathrm{X}$ & - & - & $\mathrm{x}$ \\
\hline Anartia jatrophae jatrophae (LINNAEUS, 1763) & - & - & $\mathrm{X}$ & $\mathrm{X}$ & - & - & $\mathrm{X}$ & - & - & - & $\mathrm{X}$ \\
\hline Chlosyne lacinia saundersi (DOUBLEDAY, [1847]) & $\mathrm{X}$ & - & $\mathrm{X}$ & $\mathrm{X}$ & - & - & - & - & - & - & $\mathrm{x}$ \\
\hline Colobura dirce dirce (LINNAEUS, 1758) & - & - & - & - & - & $\mathrm{x}$ & - & - & - & - & - \\
\hline Eresia Lansdorfi (GODART, 1819) & - & - & - & $\mathrm{x}$ & - & $x$ & - & $\mathrm{x}$ & - & - & $\mathrm{x}$ \\
\hline Hypanartia lethe lethe (FABRICIUS, 1793) & $\mathrm{X}$ & - & - & $\mathrm{X}$ & - & - & - & $\mathrm{X}$ & - & - & - \\
\hline Junonia evarete evarete (CRAMER, 1779) & - & $\mathrm{X}$ & $\mathrm{X}$ & $\mathrm{x}$ & - & $\mathrm{X}$ & $\mathrm{X}$ & - & - & - & $\mathrm{x}$ \\
\hline Ortilia dicoma (HEWITSON, 1864) & - & - & - & $\mathrm{x}$ & - & - & - & - & - & - & - \\
\hline Ortilia gentina (HIGGINS, 1981) & - & - & - & - & - & - & $\mathrm{X}$ & - & - & - & - \\
\hline Ortilia ithra (KIRBY, 1900) & $\mathrm{x}$ & - & $\mathrm{X}$ & $\mathrm{x}$ & - & - & - & - & - & - & $\mathrm{x}$ \\
\hline Ortilia orthia (HEWITSON, 1864) & - & - & $\mathrm{X}$ & $\mathrm{x}$ & - & - & - & - & - & - & $\mathrm{x}$ \\
\hline Siproeta epaphus trayja (HÜBNER, [1823]) & $\mathrm{X}$ & - & - & - & - & - & - & - & - & - & - \\
\hline Siproeta stelenes meridionalis (FRUHSTORFER, 1909) & $\mathrm{x}$ & - & - & - & - & - & $\mathrm{X}$ & - & - & - & - \\
\hline Tegosa claudina (ESCHSCHOLTZ, 1821) & $\mathrm{X}$ & - & - & $\mathrm{X}$ & - & - & - & $\mathrm{X}$ & - & $\mathrm{X}$ & - \\
\hline Telenassa teletusa teletusa (GODART, [1824]) & - & - & - & - & - & - & - & - & - & - & $\mathrm{x}$ \\
\hline
\end{tabular}




\begin{tabular}{|c|c|c|c|c|c|c|c|c|c|c|c|}
\hline Vanessa braziliensis (MOORE, 1883) & $\mathrm{X}$ & - & $\mathrm{X}$ & $x$ & - & - & - & $\mathrm{x}$ & - & - & $\mathrm{x}$ \\
\hline Vanessa myrinna (DOUBLEDAY, 1849) & - & - & $\mathrm{X}$ & $\mathrm{x}$ & - & - & - & - & - & - & $\mathrm{x}$ \\
\hline \multicolumn{12}{|l|}{ Satyrinae (32) } \\
\hline Blepolenis batea (HÜBNER, [1821]) & - & - & $x$ & - & - & - & - & - & - & - & $\mathrm{x}$ \\
\hline Brassolis astyra astyra (GODART, [1824]) & - & - & - & $\mathrm{x}$ & - & - & - & $\mathrm{x}$ & - & - & - \\
\hline Caligo illioneus illioneus (CRAMER, 1775) & - & - & - & $\mathrm{x}$ & - & - & - & - & - & - & - \\
\hline Capronnieria galesus (GODART, [1824]) & - & - & $\mathrm{X}$ & $\mathrm{x}$ & - & - & - & - & - & - & $\mathrm{X}$ \\
\hline Carminda paeon (GODART, [1824]) & - & - & $\mathrm{X}$ & $\mathrm{x}$ & - & - & - & $\mathrm{x}$ & - & - & - \\
\hline Cissia eous (BUTLER, 1867) & - & - & $\mathrm{X}$ & $x$ & - & - & - & - & - & - & $\mathrm{X}$ \\
\hline Cissia phronius (GODART, [1824]) & $\mathrm{X}$ & - & $\mathrm{X}$ & $\mathrm{x}$ & - & - & - & - & - & - & - \\
\hline Eryphanis reevesii reevesii (DOUBLEDAY, [1849]) & - & - & - & $\mathrm{x}$ & - & - & - & $x$ & - & - & - \\
\hline Euptychoides castrensis (SCHAUS, [1902]) & - & - & $\mathrm{x}$ & - & - & - & - & - & - & - & - \\
\hline Forsterinaria necys (GODART, [1824]) & - & - & $\mathrm{x}$ & - & - & - & - & - & - & - & $\mathrm{x}$ \\
\hline Forsterinaria pronophila (BUTLER, 1867) & - & - & $\mathrm{X}$ & - & - & - & - & - & - & - & - \\
\hline Godartiana muscosa (BUTLER, 1870) & - & - & $\mathrm{x}$ & $x$ & - & - & - & $\mathrm{x}$ & - & - & $\mathrm{X}$ \\
\hline Hermeuptychia sp. & $\mathrm{X}$ & - & $\mathrm{X}$ & $\mathrm{x}$ & - & - & - & $\mathrm{x}$ & - & - & $\mathrm{x}$ \\
\hline Moneuptychia soter (BUTLER, 1877) & - & - & - & - & - & - & $\mathrm{x}$ & $\mathrm{x}$ & - & - & $\mathrm{X}$ \\
\hline Moneuptychia sp. & - & - & $\mathrm{X}$ & - & - & - & - & - & - & - & - \\
\hline Morpho helenor achillides (FELDER \& FELDER, 1867) & $\mathrm{x}$ & - & $\mathrm{X}$ & $x$ & $\mathrm{x}$ & $\mathrm{x}$ & $\mathrm{x}$ & $\mathrm{x}$ & - & - & $\mathrm{x}$ \\
\hline Morpho iphitus iphitus (FELDER \& FELDER, 1867) & - & - & $\mathrm{X}$ & - & - & - & - & - & - & $\mathrm{x}$ & $\mathrm{X}$ \\
\hline Morpho menelaus coeruleus (PERRY, 1810) & - & - & - & - & - & - & - & - & - & - & $\mathrm{X}$ \\
\hline Opsiphanes cassiae (LINNAEUS, 1758) & $\mathrm{X}$ & - & - & - & - & - & - & - & $\mathrm{x}$ & - & - \\
\hline Pareuptychia ocirrhoe interjecta (D'ALMEIDA, 1952) & $\mathrm{X}$ & - & - & $\mathrm{x}$ & - & - & - & - & - & - & $\mathrm{X}$ \\
\hline Paryphthimoides vestigiata (BUTLER, 1867) & $\mathrm{X}$ & - & - & - & - & - & $\mathrm{x}$ & - & - & - & $\mathrm{x}$ \\
\hline Pharneuptychia sp. & $\mathrm{X}$ & - & - & $\mathrm{x}$ & - & - & - & - & - & - & - \\
\hline Pseudodebis ypthima (HÜBNER, [1821]) & - & - & - & - & - & - & - & - & - & - & $\mathrm{X}$ \\
\hline Stegosatyrus ocelloides (SCHAUS, 1902) & - & - & - & - & - & - & - & - & - & - & $\mathrm{X}$ \\
\hline Taygetis sp. & $\mathrm{X}$ & - & - & $x$ & - & - & - & - & - & - & - \\
\hline Taygetis tripunctata (WEYMER, 1907) & - & - & - & $x$ & - & - & - & - & - & - & - \\
\hline Taygetis virgilia (CRAMER, 1776) & $\mathrm{X}$ & - & - & $\mathrm{x}$ & - & - & - & - & - & - & - \\
\hline Yphthimoides affinis (BUTLER, 1867) & - & - & - & - & - & - & $\mathrm{x}$ & - & - & - & - \\
\hline Yphthimoides angularis (BUTLER, 1867) & $\mathrm{X}$ & - & - & - & - & - & - & - & - & - & - \\
\hline Yphthimoides straminea (BUTLER, 1867) & - & - & - & $x$ & - & - & $\mathrm{x}$ & - & - & - & - \\
\hline Yphthimoides viviana (ROMIEUX, 1927) & - & - & $\mathrm{X}$ & - & - & - & - & - & - & - & - \\
\hline Yphthimoides yphthima (FELDER \& FELDER, 1867) & - & - & - & - & - & - & $\mathrm{x}$ & - & - & - & - \\
\hline \multicolumn{12}{|l|}{ PAPILIONIDAE (12) } \\
\hline \multicolumn{12}{|l|}{ Papilioninae (12) } \\
\hline Battus polydamas polydamas (LINNAEUS, 1758) & - & - & - & $\mathrm{x}$ & - & - & - & - & - & - & - \\
\hline Heraclides anchisiades capys (HÜBNER, [1809]) & - & - & - & - & - & - & - & - & - & $\mathrm{x}$ & - \\
\hline Heraclides androgeus laodocus (FABRICIUS, 1793) & - & - & - & - & - & - & - & - & - & - & $\mathrm{X}$ \\
\hline Heraclides astyalus astyalus (GODART, 1819) & $\mathrm{X}$ & - & - & $x$ & - & - & - & - & - & - & $\mathrm{X}$ \\
\hline Heraclides hectorides (ESPER, 1794) & - & - & $\mathrm{X}$ & - & $\mathrm{x}$ & - & - & $\mathrm{x}$ & - & - & $\mathrm{X}$ \\
\hline Mimoides lysithous lysithous (HÜBNER, [1821]) & - & - & $\mathrm{x}$ & - & - & - & - & - & - & - & $\mathrm{x}$ \\
\hline Parides agavus (DRURY, 1782) & $\mathrm{x}$ & - & - & $x$ & - & - & - & $x$ & - & - & - \\
\hline Parides anchises nephalion (GODART, 1819) & - & - & $\mathrm{X}$ & - & - & - & $\mathrm{x}$ & - & - & $\mathrm{X}$ & $\mathrm{x}$ \\
\hline Parides bunichus bunichus (HÜBNER, [1821]) & - & - & $\mathrm{X}$ & $x$ & - & - & - & - & - & - & $\mathrm{X}$ \\
\hline Parides neophilus eurybates (GRAY, [1853]) & - & - & $\mathrm{X}$ & - & - & - & - & - & - & - & $\mathrm{X}$ \\
\hline Parides proneus (HÜBNER, [1831]) & - & - & $\mathrm{X}$ & $\mathrm{x}$ & - & - & $\mathrm{x}$ & - & - & - & $\mathrm{x}$ \\
\hline Pterourus scamander grayi (BOISDUVAL, 1836) & - & - & - & $\mathrm{x}$ & - & $\mathrm{x}$ & - & - & - & - & - \\
\hline \multicolumn{12}{|l|}{ PIERIDAE (22) } \\
\hline \multicolumn{12}{|l|}{ Coliadinae (12) } \\
\hline Eurema agave pallida (CHAVANNES, 1850) & - & - & $\mathrm{X}$ & $\mathrm{x}$ & - & - & - & & - & - & $\mathrm{X}$ \\
\hline Eurema albula sinoe (GODART, 1819) & $\mathrm{X}$ & $\mathrm{X}$ & $\mathrm{X}$ & $\mathrm{X}$ & - & - & $\mathrm{X}$ & $\mathrm{X}$ & - & $\mathrm{X}$ & $\mathrm{X}$ \\
\hline Eurema arbela (GEYER, 1832) & - & - & - & $\mathrm{x}$ & - & - & - & - & - & - & - \\
\hline Eurema deva deva (DOUBLEDAY, 1847) & - & - & $\mathrm{x}$ & $\mathrm{x}$ & - & - & - & $\mathrm{x}$ & - & - & $\mathrm{x}$ \\
\hline Eurema elathea flavescens (CHAVANNES, 1850) & $\mathrm{x}$ & - & $\mathrm{x}$ & $\mathrm{x}$ & - & - & $\mathrm{x}$ & $\mathrm{x}$ & - & - & $\mathrm{x}$ \\
\hline Eurema phiale paula (RÖBER, 1909) & - & - & $\mathrm{X}$ & $\mathrm{x}$ & - & $\mathrm{x}$ & - & $\mathrm{X}$ & $\mathrm{x}$ & - & $\mathrm{X}$ \\
\hline Phoebis argante argante (FABRICIUS, 1775) & - & - & - & $\mathrm{X}$ & - & - & - & - & - & - & - \\
\hline Phoebis neocypris neocypris (HÜBNER, [1823]) & - & - & - & $\mathrm{x}$ & - & - & - & - & - & - & $\mathrm{x}$ \\
\hline Phoebis philea philea (LINNAEUS, 1763) & $\mathrm{X}$ & - & - & - & - & - & - & $\mathrm{X}$ & - & - & - \\
\hline Phoebis sennae marcellina (CRAMER, 1777) & $\mathrm{X}$ & - & - & $\mathrm{x}$ & - & - & - & - & - & - & $\mathrm{x}$ \\
\hline Pyrisitia leuce leuce (BOISDUVAL, 1836) & - & - & - & $\mathrm{x}$ & - & - & - & - & - & $\mathrm{x}$ & $\mathrm{X}$ \\
\hline Pyrisitia nise tenella (BOISDUVAL, 1836) & $\mathrm{x}$ & - & $\mathrm{x}$ & $\mathrm{x}$ & - & - & $\mathrm{x}$ & $\mathrm{x}$ & - & - & $\mathrm{x}$ \\
\hline \multicolumn{12}{|l|}{ Dismorphiinae (3) } \\
\hline Dismorphia amphione astynome (DALMAN, 1823) & - & - & - & - & - & - & $\mathrm{x}$ & - & - & - & - \\
\hline Dismorphia thermesia thermesia (GODART, 1819) & - & - & $\mathrm{X}$ & - & - & - & - & - & - & - & - \\
\hline
\end{tabular}




\begin{tabular}{|c|c|c|c|c|c|c|c|c|c|c|c|}
\hline Pseudopieris nehemia nehemia (BOISDUVAL, 1836) & - & - & $\mathrm{X}$ & $\mathrm{X}$ & - & - & - & - & - & - & $\mathrm{X}$ \\
\hline \multicolumn{12}{|l|}{ Pierinae (7) } \\
\hline Archonias brassolis tereas (GODART, 1819) & - & - & $\mathrm{X}$ & - & - & - & - & $\mathrm{X}$ & - & - & $\mathrm{X}$ \\
\hline Ascia monuste orseis (GODART, 1819) & - & - & $\mathrm{X}$ & $\mathrm{X}$ & - & - & $\mathrm{X}$ & $\mathrm{X}$ & - & - & $\mathrm{X}$ \\
\hline Charonias theano (BOISDUVAL, 1836) & - & - & - & - & - & - & - & - & - & - & $\mathrm{X}$ \\
\hline Melete lycimnia (CRAMER, 1777) & - & - & - & $\mathrm{X}$ & - & - & - & - & - & - & $\mathrm{X}$ \\
\hline Pereute antodyca (BOISDUVAL, 1836) & - & - & $\mathrm{x}$ & - & - & - & - & - & - & - & - \\
\hline Pereute swainsoni (GRAY, 1832) & - & - & - & - & - & - & - & $X$ & - & - & - \\
\hline Theochila maenacte itatiayae (FOETTERLE, 1902) & - & - & $\mathrm{X}$ & - & - & - & - & $\mathrm{X}$ & - & - & $\mathrm{X}$ \\
\hline \multicolumn{12}{|l|}{ RIODINIDAE (24) } \\
\hline \multicolumn{12}{|l|}{ Nemeobiinae (1) } \\
\hline Euselasia zara (WESTWOOD, 1851) & - & - & - & - & - & - & - & - & - & - & $\mathrm{X}$ \\
\hline \multicolumn{12}{|l|}{ Riodininae (23) } \\
\hline Anteros lectabilis (STICHEL, 1909) & - & - & - & - & - & - & - & - & - & - & $X$ \\
\hline Aricoris constantius (FABRICIUS, 1793) & - & - & - & - & - & - & - & $\mathrm{X}$ & - & - & - \\
\hline Baeotis johannae johannae (SHARPE, [1890]) & $\mathrm{X}$ & - & - & $\mathrm{X}$ & - & - & - & - & - & - & - \\
\hline Barbicornis basilis GODART, [1824] & - & - & - & $\mathrm{X}$ & - & - & - & - & - & - & - \\
\hline Calephelis braziliensis MCALPINE, 1971 & - & - & - & $\mathrm{X}$ & - & - & - & $\mathrm{X}$ & - & - & $X$ \\
\hline Caria sp. & - & - & - & - & - & - & - & $\mathrm{X}$ & - & - & - \\
\hline Chalodeta theodora (FELDER \& FELDER 1862) & - & - & - & $\mathrm{X}$ & - & - & - & - & - & - & - \\
\hline Charis cadytis (HEWITSON, 1866) & $\mathrm{X}$ & - & - & - & - & - & - & - & - & - & $\mathrm{X}$ \\
\hline Chorinea heliconides (SWAINSON, 1833) & - & - & - & - & - & - & - & - & - & - & $\mathrm{x}$ \\
\hline Detritivora sp. & - & - & - & $\mathrm{X}$ & - & $\mathrm{X}$ & - & - & - & - & - \\
\hline Emesis fastidiosa MÉNÉTRIÉS, 1855 & - & - & - & - & - & - & - & - & - & - & $\mathrm{x}$ \\
\hline Emesis sp. & - & - & - & $\mathrm{X}$ & - & - & - & - & - & - & - \\
\hline Eurybia pergaea (GEYER, 1832) & - & - & $\mathrm{X}$ & - & - & - & - & - & - & - & $\mathrm{X}$ \\
\hline Lasaia agesilas agesilas (LATREILLE, [1809]) & - & - & - & - & - & - & - & - & - & - & $\mathrm{X}$ \\
\hline Lemonias zygia zygia (HÜBNER, [1807]) & - & - & - & $\mathrm{X}$ & $\mathrm{X}$ & $\mathrm{X}$ & - & $\mathrm{X}$ & - & - & $X$ \\
\hline Melanis sp. & - & - & - & - & - & - & $\mathrm{X}$ & - & - & - & $\mathrm{X}$ \\
\hline Panara jarbas thymele (STICHEL, 1909) & $\mathrm{X}$ & - & - & - & - & - & - & - & - & - & - \\
\hline Panara soana bacana CALLAGHAN, 1997 & - & - & - & $\mathrm{X}$ & - & - & - & - & - & - & $\mathrm{X}$ \\
\hline Rhetus periander eleusinus (STICHEL, 1910) & - & - & - & - & - & - & - & - & - & - & $\mathrm{X}$ \\
\hline Riodina lycisca lycisca (HEWITSON, [1853]) & - & - & - & $\mathrm{X}$ & - & - & - & - & - & - & $\mathrm{X}$ \\
\hline Stichelia bocchoris (HEWITSON, 1876) & - & - & $\mathrm{X}$ & - & - & - & - & - & - & - & - \\
\hline Synargis calyce (FELDER \& FELDER, 1862) & $\mathrm{X}$ & - & - & - & - & - & - & - & - & - & - \\
\hline Synargis paulistina (STICHEL, 1910) & - & - & - & - & - & - & - & - & - & - & $\mathrm{X}$ \\
\hline Total de espécies por localidades & 67 & 3 & 82 & 131 & 6 & 12 & 47 & 61 & 4 & 14 & 126 \\
\hline
\end{tabular}

O estimador de espécies Jackinife 1 mostrou que o número de espécies pode chegar a 295 (Figura 4), uma eficiência de coleta em torno de $78 \%$.

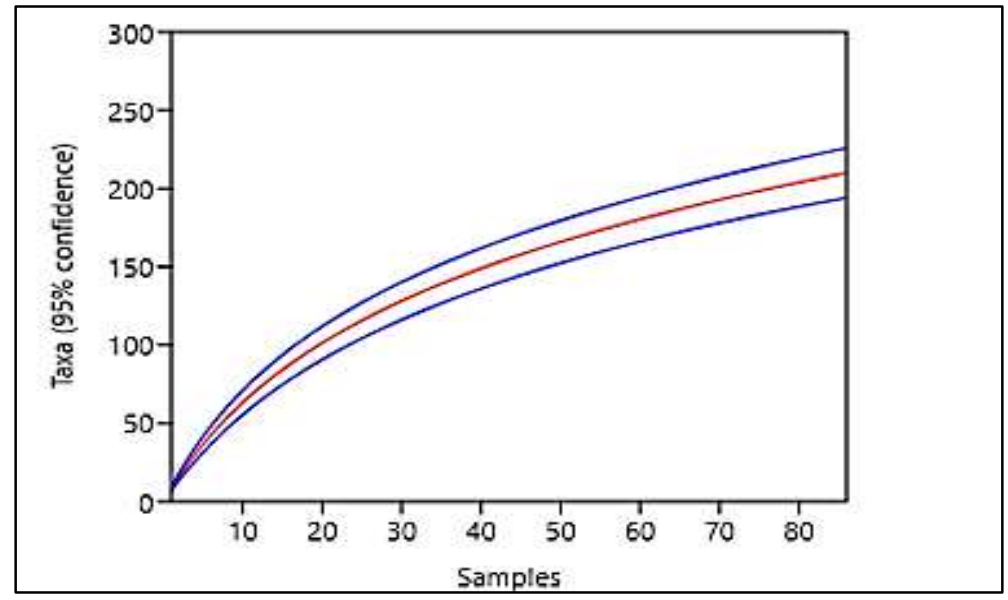

Figura 3: Número de espécies de borboletas estimadas (estimador de riqueza Jackinife) e número de espécies coletadas em 105 dias no sul de Minas Gerais.

\section{DISCUSSÃO}

Quando comparado com outros trabalhos realizados na mesma fitofisionomia, o presente estudo 
apresentou um maior número de espécies (SILVA et al., 2007; SILVA et al., 2017), o que pode ser reflexo do maior esforço amostral. A família Nymphalidae foi a mais rica em função de diferentes fatores, como por exemplo, por serem encontradas em diferentes ecossistemas (DEVRIES, 1987) e pela grande diversidade de nichos alimentares (BROWN JUNIOR et al., 1999). Esse padrão também foi registrado em outros estudos, como Oliveira et al. (2018) que registrou 57\% de ninfalídeos, Andrade et al. (2017) com 73,56\%, Silva et al. (2007) com 46,1\% e Araújo et al. (2015) com 73,9\%.

A subfamília Satyrinae forma a maior subfamília de Nymphalidae em número de espécies, são frugívoras e comumente usam espécies de Poaceae (gramíneas) como plantas hospedeiras (BECCALONI et al., 2008, MARín et al., 2011). Como na área de estudo há muitos campos e áreas de pastagem, isso explicaria o resultado observado, como já elucidado em outros trabalhos realizado em outras fitofisionomias no Brasil (AOKI et al., 2012; MORAES et al., 2012).

A subespécie Mechanitis polymnia casabranca também foi registrada como a mais abundante nos trabalhos de Araújo et al. (2015), em um Parque Ecológico de perímetro urbano, Andrade et al. (2017) em um fragmento de Mata Atlântica. Essa espécie é considerada comum em florestas tropicais, fragmentos urbanos, ambientes perturbados e matas secundárias em formação (DEVRIES, 1987; BROWN JUNIOR, 1992; BROWN JUNIOR et al., 2003). É também comumente encontrada associada a plantas da família Solanaceae, podendo ser considerada uma praga importante (TAVARES et al., 2014; LORDELLO, 1951).

A frequência da espécie Tegosa claudina pode ser resultado de sua interação com as inflorescências das plantas Wedelia paludosa, Bidens pilosa, Bidens alba, todas da família Asteraceae, que têm floração durante o ano todo (HURTADO et al., 2009), e ocorrem comumente em campos naturais, áreas de pastagem e antropizadas, ecossistemas comuns na área de estudo. Segundo Freitas (1991) T. claudina é frequentemente encontrada no sul e sudeste do Brasil. A espécie foi abundante em outros estudos (CARVALHO et al., 2015; MORAIS et al., 2012; SILVA et al., 2017), porém não foi citada sua frequência.

Um interessante registro, foi o de Charonias theano, uma das borboletas que compõem a lista das espécies ameaçadas do Brasil (ICMBIO, 2018), encontrada em área de floresta ripária do rio Machado no município de Poço Fundo. A distribuição dessa espécie pode ser encontrada em Freitas et al., (2011), associada a floresta semidecidual submontana e montana (500-1.100 m) nos seguintes estados: Paraná e Santa Catarina (DOLIBAINA et al., 2011), Minas Gerais e São Paulo (EBERT, 1969; ZIKÁN et al., 1968), Rio de Janeiro (MONTEIRO et al., 2010) e também Espírito Santo (BROWN et al., 2000). Seus registros para os municípios de Minas Gerais são: Caxambu, Passa Quatro, Pouso Alegre, Poços de Caldas, Cambuquira e Conceição dos Ouros (FREITAS et al., 2011) e um recente no Parque Estadual da Serra do Papagaio (OLIVEIRA et al., 2018). Essa espécie também foi redescoberta no estado de São Paulo em Jundiaí, e apesar dessa área ter sido intensivamente inventariada desde o início dos anos 1990, somente em 2011 ela foi encontrada (BROWN JUNIOR, 1992; FREITAS et al., 2011), demonstrando assim a importância da continuidade de inventários mesmo em regiões com intenso esforço amostral.

Considerando que o esforço amostral na APA do Rio Machado tenha sido menor quando comparado aos municípios de Bueno Brandão e Inconfidentes (Tabela 1), foi obtido uma riqueza 
significativa de espécies de borboletas, o que mostra a importância da conservação desta Unidade de Conservação. $O$ estimador de espécies mostrou que um maior esforço de amostragem permitiria registrar mais espécies, próximo do esperado. A eficiência também poderia ser maior se fossem utilizadas as armadilhas atrativas do tipo Van Someren-Rydon (RYDON, 1964), que auxiliam na coleta de espécies frugívoras da família Nymphalidae (DEVRIES, 1987; FREITAS et al., 2003; UEHARA-PRADO et al., 2007).

\section{CONCLUSÕES}

A região do sul de Minas Gerais abriga uma importante fauna de borboletas para o Brasil, com ênfase na APA do Rio Machado, que possui a maior riqueza das áreas amostradas e pela presença da espécie ameaçada de extinção Charonias theano, mas que sofre diferentes ações antrópicas.

AGRADECIMENTOS: Ao Laboratório de Ecologia e Sistemática de Borboletas (LABBOR); ao Laboratório de Zoologia do IFSULDEMINAS, campus Inconfidentes; ao SISBIO; ao IEF-MG; ao IFSULDEMIMNAS, Campus Machado e Inconfidentes; a Coordenação de Aperfeiçoamento de Pessoal de Nível Superior (CAPES), Código de financiamento 001.

\section{REFERÊNCIAS}

ANDRADE, D. A.; TEIXEIRA, I. R. V.. Diversidade de Lepidoptera em um fragmento florestal em Muzambinho, Minas Gerais. Ciência Florestal, Santa Maria, v.27, n.4, p.1229-1241, 2017. DOI: https://doi.org/10.5902/1980509830311

AOKI, C.; PIRES, A. C. V.; HOKAMA, D.; TEIXEIRA-GAMARRA, M. C.. Borboletas da Reserva Particular do Patrimônio Natural Engenheiro Eliezer Batista. In: RABELO, A. P. C., MOREIRA, V. F., BERTASSONI, A.; AOKI, C.. Descobrindo o Paraíso: Aspectos Biológicos da Reserva Particular do Patrimônio Natural Engenheiro Eliezer Batista. Rio de Janeiro: Instituto Homem Pantaneiro, 2012. p.154-169.

ARAÚJO, M. C.; PAPROCKI, H.. Lista de Lepidoptera do Parque Ecológico Felisberto Neves, Betim, Minas Gerais. Sinapse Múltipla, v.4, n.1, p.1-15, 2015.

BECCALONI, G. W.; VILORIA, A. L.; HALL, S. K.; ROBINSON, G. S.. Catalogue of the hostplants of the Neotropical butterflies. Monografías Tercer Milenio, v.8, p.1-536, 2008.

BROWN JUNIOR, K. S.; MIELKE, O. H. H.. Lepidoptera of the central Brazil plateau: I. Preliminary list of Rhopalocera: Introduction, Nymphalidae, Libytheidae. Journal of the Lepidopterists' Society, v.21, p.77-106, 1967a.

BROWN JUNIOR, K. S.; MIELKE, O. H. H.. Lepidoptera of the central Brazil plateau: II. Preliminary list of Rhopalocera: Lycaenidae, Pieridae, Papilionidae, Hesperiidae. Journal of the Lepidopterists' Society v.21, p.145-168, 1967b.

BROWN JUNIOR, K. S.; MIELKE, O. H. H.. Lepidoptera of the central Brazil plateau: III. Partiallist for the Belo Horizonte area, showing the character of the southeastern "blend zone". Journal of the Lepidopterists' Society, v.22, p.147$157,1968$.
BROWN JUNIOR, K. S.. Maximizing daily butterfly counts. Journal of the Lepidopterists' Society, v.26, n.3, p.183-196. 1972a.

BROWN JUNIOR, K. S.. Borboletas da Serra do Japi: diversidade, habitats, recursos alimentares e variação temporal In: MORELLATO, L. P. C.. História Natural da Serra do Japi: ecologia e preservação de uma área florestal do Sudeste do Brasil. Campinas: UNICAMP, 1992. p.142-186.

BROWN JUNIOR, K. S.; FREITAS, A. V. L.. Lepidoptera. In SIMONE, L.; ISMAEL, D.; VALENTI, W.; MATSUMURATUNDISI, T.; ROCHA, O.; SIMONE, L.; VALENTI, W. C.; TUNDISI, T. M.; ROCHA, R. O.. Biodiversidade do Estado de São Paulo: síntese do conhecimento ao final do século XX. 5 ed. 1999. p.225-243.

BROWN JUNIOR, K. S.; FREITAS, A. V. L.. Diversidade de Lepidoptera em Santa Teresa, Espírito Santo. Boletim do Museu de Biologia Mello Leitão, Santa Teresa, v.11, n.12, p.71-116, 2000.

BROWN JUNIOR, K. S.; FREITAS, A. V. L.. Comunidades de borboletas de fragmentos urbanos florestais em Campinas, São Paulo, Brasil: estrutura, instabilidade, correlatos ambientais e de conservação. Journal of Conservation Insect, Headington, v.6, n.4, p.217-231, 2003.

CARVALHO, A. P. S.; MORAIS, A. B. B.. Borboletas associadas a ambientes de restinga no extremo sul do Brasil (Lepidoptera: Papilionoidea). SHILAP Revista de Lepidopterologia, v.43, p.349-363, 2015.

CASAGRANDE, M. M.; MIELKE, O. H. H.; BROWN JR, K. S. Borboletas (LEPIDOPTERA) ameaçadas de extinção em Minas Gerais, Brasil. Revista brasileira de Zoologia, Curitiba, v.15, n.1, p.241-259, 1998. DOI: https://doi.org/10.1590/S0101$\underline{81751998000100021}$ 
CASAGRANDE, M. M.; M. Duarte.. Lepidoptera in Catálogo Taxonômico da Fauna do Brasil. PNUD, 2019.

COPAM. Conselho Estadual de Política Ambiental. Deliberação Normativa COPAM n.147, de 30 de abril de 2010. Aprova a Lista de Espécies Ameaçadas de Extinção da Fauna do Estado de Minas Gerais. Belo Horizonte: Diário do Executivo do Estado de Minas Gerais, 2010.

DEVRIES, P. J.. The butterflies of Costa Rica and their natural history. Papilionidae, Pieridae and Nymphalidae. New Jersey: Priceton University, 1987.

DOLIBAINA, D. R.; MIELKE O. H. H.; CASAGRANDE, M. M.. Borboletas (Papilionoidea e Hesperioidea) de Guarapuava e arredores, Paraná, Brasil: um inventário com base em 63 anos de registros. Biota Neotropica, Campinas, v.11, n.1, p.341-354, 2011. DOI: https://doi.org/10.1590/S167606032011000100031

EBERT, H.. On the frequency of butterfl ies in eastern Brazil, with a list of the butterfl y fauna of Poços de Caldas, Minas Gerais. Journal of the Lepidopterists' Society, v.23, p.1-48, 1969.

FREITAS, A. V. L.; FRANCINI, R. B.; BROWN JR, K. S.. Insetos como indicadores ambientais. In: CULLEN JUNIOR, L.; RUDRAN, R.; VALLADARES-PÁDUA, C.. Métodos de estudo em Biologia da Conservação e Manejo da Vida Silvestre. 2 ed. Curitiba: UFPR, 2003. p.125-151.

FREITAS, A. V. L.; LEAL, I. R.; UEHARA-PRADO, M.; IANNUZZI, L.. Insetos como indicadores de conservação da paisagem. In: ROCHA, C. F. D.; BERGALLO, H. G.; VAN SLUYS, M.; ALVES, M. A. S.. Biologia da conservação. Rio de Janeiro: UERJ, 2006. p.201-225.

FREITAS, A. V. L.; KAMINSKI, L. A.; ISERHARD, C. A.; BARBOSA, E. P.; MARINI FILHO, O. J.. The endangered butterfly Charonias theano (BOISDUVAL, 1836) (Lepidoptera: Pieridae): current status, threats and its rediscovery in the state of São Paulo, southeastern Brazil. Neotropical Entomology, Londrina, v.40, n.6, p.669-676, 2011. DOI: http://dx.doi.org/10.1590/S1519-566X2011000600006

HENRIQUES, N. R.; BEIRÃO, M. D. V.; BRASIL, E.; CORNELISSEN, T.. Butterflies (Lepidoptera: Papilionoidea) from the campos rupestres of Serra de São José, Minas Gerais, Brazil. Biota Neotropica, Campinas, v.19, n.3, 2019. DOI: https://doi.org/10.1590/1676-0611-bn-2018-0655

HURTADO, J.P.; FRANCINI, R. B.. Como a borboleta Tegosa claudina (Nymphalidae: melitaeinae) forrageia?. In: CONGRESSO DE ECOLOGIA DO BRASIL, 9. Anais. São Lourenço, 2009.

ICMBIO. Instituto Chico Mendes de Conservação da Biodiversidade. Livro Vermelho da Fauna Brasileira Ameaçada de Extinção: Volume VII: Invertebrados. Brasília: ICMBio, 2018.

LAMAS, G.. Checklist: Part 4A. Hesperioidea-Papilionoidea. In: HEPPNER, J. B.. Atlas of Neotropical Lepidoptera. Gainesville: Association for Tropical Lepidoptera, 2004. p.1439.

LOMOV, B.; KEITH, D. A.; BRITTON, D. R.; HOCHULI, D. F.. Are butterflies and moths useful indicators for restoration monitoring? A pilot study in Sydney's Cumberland Plain Woodland. Ecological Management and Restoration, v.7, n.3, p.204-210, 2006. DOI: https://doi.org/10.1111/j.14428903.2006.00310.x

LORDELLO, L. G. E.. Contribuição ao conhecimento de alguns Ithomiidae brasileiros, predadores de Solanáceas (Lep., Rhopalocera). Anais da Escola Superior de Agricultura Luiz de Queiroz, Piracicaba, v.8, p.99-110, 1951. DOI: http://dx.doi.org/10.1590/S0071-12761951000100006

MARÍN, M. A.; PEÑA, C.; FREITAS, A. V. L.; WAHLBERG, N.; URIBE, S. I.. From the phylogeny of the Satyrinae butterflies to the systematics of Euptychiina (Lepidoptera: Nymphalidae): history, progress and prospects. Neotropical Entomology, Londrina, v.40, n.1, 2011. DOI: https://doi.org/10.1590/S1519-566X2011000100001

MIELKE, O. H. H.. Catalogue of the American Hesperioidea: Hesperiidae. Hesperiinae 1: Adlerodea, Lychnuchus, Curitiba: Sociedade Brasileira de Zoologia, 2005a.

MIELKE, O. H. H.. Catalogue of the American Hesperioidea: Hesperiidae. Hesperiinae 2: Megaleas, Zenis, Curitiba: Sociedade Brasileira de Zoologia, 2005b.

MIELKE, O. H. H.. Catalogue of the American Hesperioidea: Hesperiidae: Pyrhopyginae. Complementary and supplementary parts to the Checklist of the Neotropical Region. Curitiba: Sociedade Brasileira de Zoologia, 2014a.

MIELKE, O. H. H.. Catalogue of the American Hesperioidea: Hesperiidae. Pyrginae 1: Eudamini. Curitiba: Sociedade Brasileira de Zoologia, 2014b.

MIELKE, O. H. H.. Catalogue of the American Hesperioidea: Hesperiidae. Volume 3. Pyrginae 2: Pyrgini. Curitiba: Sociedade Brasileira de Zoologia, 2014c.

MITTER, C.; DAVIS, D. R.; CUMMINGS, M. P.. Phylogeny and evolution of Lepidoptera. Annual Review of Entomology, v.62, p.265-283, 2017. DOI:

https://doi.org/10.1146/annurev-ento-031616-035125

MONTEIRO, R. F.; FREITAS, A. V. L.; COSTA FILHO, M. A. D. F.; NASCIMENTO, M. D. S.; ALVES, T. G.; BROWN JR, K. S.; MIELKE, O. H. H.; CASAGRANDE, M. M.; DUARTE, M.. Borboletas da Mata Atlântica do estado do Rio de Janeiro: Pieridae (Lepidoptera). Arquivos do Museu Nacional, Rio de Janeiro, v.67, n.3-4, p.283-289, 2009.

MORAIS, A. B. B. D.; LEMES, R.; RITTER, C. D.. Borboletas (Lepidoptera: Hesperioidea e Papilionoidea) de Val de Serra, região central do Rio Grande do Sul, Brasil. Biota Neotropica, Campinas, v.12, n.2, p.175-183, 2012. DOI: https://doi.org/10.1590/S1676-06032012000200017

NERY, I.; CARVALHO, N.; PAPROCKI, H.. Checklist of butterflies (Insecta: Lepidoptera) from Serra do Intendente State Park-Minas Gerais, Brazil. Biodiversity Data Journal, n.2, 2014. DOI: https://doi.org/10.3897/BDJ.2.e3999

OLIVEIRA FILHO, A. T.. Definição e delimitação de domínios e subdomínios das paisagens naturais do estado de Minas Gerais. In: SCOLFORO, J. R.; CARVALHO, L. M. T..

Mapeamento e Inventário da Flora e dos Reflorestamentos de Minas Gerais. Lavras: UFLA, 2006. p.21-35.

OLIVEIRA, L. A.; MILANI, L. R.; SOUZA M. M.. Riqueza de 
borboletas (Lepidoptera) no Parque Estadual da Serra do Papagaio, sul de Minas Gerais, Brasil. MG Biota, Belo Horizonte, v.11, n.3, 2018.

PIRES, A. C. V.; BEIRÃO, M. V.; FERNANDES, G. W.; OLIVEIRA, I. F.; PEREIRA, G. C. N.; SILVA, V. D.; MIELKE, O. H. H.; DUARTE, M.. Checklist of butterflies from the rupestrian grasslands of Serra do Cipó, Minas Gerais, Brazil (Lepidoptera: Papilionoidea). SHILAP Revista de Lepidopterología, v.46, n.181, p.5-17, 2018.

RYDON, A.. Notes on the use of butterfly traps in East Africa. Journal of the Lepidopterists' Society, v.18, n.1, p.51-58, 1964.

SERAPHIM, N.; KAMINSKI, L. A.; DEVRIES, P. J.; PENZ, C.; CALLAGHAN, C.; WAHLBERG, N.; SILVA-BRANDÃO, K. L.; FREITAS, A. V.. Molecular phylogeny and higher systematics of the metalmark butterflies (Lepidoptera: Riodinidae). Systematic Entomology, v.43, n.2, p.407-425, 2018. DOI https://doi.org/10.1111/syen.12282

SILVA, A.R. M.; CASTRO, C. O.; MAFIA, P. O.; MENDONÇA, M O. C.; ALVES, T. C. C.; BEIRÃO, M. V.. Borboletas frugívoras (Lepidoptera: Nymphalidae) de uma área urbana (Área de Proteção Especial Manancial Cercadinho) em Belo Horizonte, Minas Gerais, Brasil. Biota Neotropica, v.12, n.3, p. 292-297, 2012.

SILVA, A. R.; LANDA, G. G.; VITALINO, R. F.. Borboletas (Lepidoptera) de um fragmento de mata urbano em Minas Gerais, Brasil. Lundiana, v.8, n.2, p.137-142, 2007.

SILVA, J. M.; GAWLINSKI, M.; MOSCOSO, M.; ZURSCHIMITEN, M. P. V.; CUNHA, S. K.; SILVA; E. J. E.; GARCIA, F. R. M.. Borboletas em Floresta Estacional Semidecidual e Campos do Bioma Pampa, Brasil (Lepidoptera: Papilionoidea). SHILAP Revista de Lepidopterología, v.45, n.179, p.357-368, 2017.

SOARES, G. R.; OLIVEIRA, A. A. P.; SILVA, A. R. M.. Borboletas (Lepidoptera: Papilionoidea e Hesperioidea) de um parque urbano em Belo Horizonte, Minas Gerais, Brasil. Biota

Neotrop, Campinas, v.12, n.4, 2012. DOI:

http://dx.doi.org/10.1590/S1676-06032012000400022

SOLDATI, D.; SILVEIRA, F. A.; SILVA, A. R. M.. Butterfly fauna (Lepidoptera, Papilionoidea) in a heterogeneous area between two biodiversity hotspots in Minas Gerais, Brazil.

Papéis Avulsos de Zoologia, v.59, 2019. DOI:

http://doi.org/10.11606/1807-0205/2019.59.02

TAVARES, W. D. S.; PEREIRA, A. I. D. A.; MIELKE, O. H. H. SERRÃO, J. E.; ZANUNCIO, J. C.. Mechanitis polymnia casabranca and Ithomia lichyi lichyi (Lepidoptera: Nymphalidae) damaging tree of Solanum granulosoleprosum (Solanaceae). CERNE, Lavras, v.20, n.1, p.165-172, 2014. DOI: http://dx.doi.org/10.1590/S010477602014000100020

UEHARA-PRADO, M.; BROWN JUNIOR, K. S.; FREITAS, A. V. L.. Species richness, composition and abundance of fruitfeeding butterflies in the Brazilian Atlantic Forest: comparison between a fragmented and a continuous landscape. Global Ecology and Biogeography, Malden, v.16, n.1, p.43-54, 2007. DOI: https://doi.org/10.1111/j.14668238.2006.00267.x

WAHLBERG, N.; LENEVEU, J.; KODANDARAMAIAH, U.; PEÑA, C.; NYLIN, S.; FREITAS, A. V. L.; BROWER, A. V. Z.. Nymphalid butterflies diversify following near demise at the Cretaceous / Tertiary boundary. Proceedings of the Royal Society of London. Bological Science, v.276, p.4295-4302, 2009. DOI: https://doi.org/10.1098/rspb.2009.1303

WARREN, A. D.; DAVIS, K. J.; STANGELAND, E. M.; PELHAM, J. P.; WILLMOTT K. R.; GRISHIN, N. V.. Illustrated Lists of American Butterflies. 2017.

ZIKÁN, J. F.; ZIKÁN, W.. Inseto-fauna do Itatiaia e da Mantiqueira III. Lepidoptera. Pesquisa Agropecuária Brasileira, v.3, n.1, p.45-109, 1968.

A CBPC - Companhia Brasileira de Produção Científica (CNPJ: 11.221.422/0001-03) detém os direitos materiais desta publicação. Os direitos referem-se à publicação do trabalho em qualquer parte do mundo, incluindo os direitos às renovações, expansões e disseminações da contribuição, bem como outros direitos subsidiários. Todos os trabalhos publicados eletronicamente poderão posteriormente ser publicados em coletâneas impressas sob coordenação da Sustenere Publishing, da Companhia Brasileira de Produção Científica e seus parceiros autorizados. Os (as) autores (as) preservam os direitos autorais, mas não têm permissão para a publicação da contribuição em outro meio, impresso ou digital, em português ou em tradução. 